\section{Amjad B. Adulghafour \\ A Department of Production Engineering and Metallurgy, University of Technology, Baghdad, Iraq Amjed_barzan@yahoo.com}

\section{Ahmed T. Hassan \\ Department of Production Engineering and Metallurgy, University of Technology/ Baghdad, Iraq Ath.Iraq@gmail.com}

Received on: 31/01/2017 Accepted on: 20/12/2017 Published online: 25/12/2018

\title{
Developing an Approach to Redesign Freeform Surfaces Using B-Spline Technique
}

\begin{abstract}
In product design process trends currently tend to use sculpture surface (freeform surface) that modeling by using (NURBS, B-spline, Bezier) technique to suit aesthetic, functional and manufacturability requirements. The B-Spline technique is one of the most important tools in computer aided geometric design (CAGD). This paper reported a new approach to design $3 D$ freeform $C A D$ model and then represented this model mathematically by using $B$-Spline technique. This mathematical represent consider one of the important aims that useful in manufacturing process through its geometric data which defines by this represent. The approach has three major steps: design $3 D$ CAD solid contain freeform surface by using CAD software, analysis and extraction the parameters including geometric data of $3 D C A D$ model based on IGES file format, and then construction the B-Spline surface of the model that contain multi patches by using basis function of the surface. The approach has been extensively tested with more than case study has 3 degrees of the surface then represented mathematically in four patches.
\end{abstract}

Keywords- 3D CAD, Freeform Surface, B-Spline, Basis Function, Multi Patches

How to cite this article: A.B. Adulghafour and A.T. Hassan, "Developing an Approach to Redesign Freeform Surfaces Using B-Spline Technique,” Engineering and Technology Journal, Vol. 36, No. 12, pp. 1213- 1221, 2018.

\section{Introduction}

Over the last few decades, three-dimensional (3D) geometric modeling has been generally used in disparate engineering fields, like Computer Aided Design and Computer Aided Manufacturing (CAD/CAM), rapid prototyping, virtual reality, etc. The requirement of geometric data processing varies from one application to another. For example, in rapid prototyping a triangulated model suffices whereas in engineering objects such as aerospace components, automobile components, injection molds, turbine blades and dies, complex surfaces are required. Due to the complexity of the shapes currently in the design of products that cannot be define using Bezier curve the B-Spline curve are used to represent these shapes. A spline is a set of curves that connected together in sequence to represent the single continuous curve. Such as a set segments of Bezier curve, connected end to end, can be called a spline curve. The difference between B-Spline curve, Hermite or Bezier curve in that B-Spline curve it usually consists of more than one curve segment. All segments are represented and affected by a set of control points, which are the coefficient of BSpline basis function polynomials. The total number of control points it has nothing to do with degree of curve, so there is a possibility to manipulate and change in shape that do not propagate beyond one or only few local segments [1].

The B-Spline curve became widely used and its play a central role in computer aided design today. These techniques consider a best tools to create new design especially in design freeform surface, The Knot values and a set of control points which represents the data structure of a B-spline curve of order $\mathrm{k}$ [2]. "The surfaces That are not regular in their shape and are made by joining different pat ches together by defining some continuity at points of joining are called the freeform surface". The functional and aesthetic are the most requirements that lead to design freeform surface. these surfaces mostly containing one or more non-quadratic, no n-planar surfaces, the CAD and other computer gr aphics software is used free-form surfacing to de scribe the skin of a 3-D geometric element, The freeform surfaces such as turbine blades, car bod ies and boat hulls, do not have radial dimensions and its unlike regular surfaces such as cylinders, co nes and spheres. [3]. The paper layout as follows: Section 2 presents the relevant research on geometric modeling technique. Section 3 describes Proposed of Developed Approach. Section 4 presents the B-Spline technique curves and surfaces. In Section 5 present the mathematical model of the non-uniform B-Spline technique as case study and their results. In Section 6 and 7 
Discussion and Conclusions from this research work.

\section{Literature Reviews}

Researchers worldwide have focused on developing various algorithms for modeling and designing surfaces and then represented these surfaces in mathematically model to obtain the data structure of these surfaces, the techniques for modeling these surfaces its Vary according to the complexity of surfaces to be processed. Literature reports very scant research work on modeling surface techniques.

Paul [4] in his research introduced a difference method in curve and surface represented depending on a little number of control points, Research discussed Bézier and B-spline curves by introducing the techniques used later.

Xiaogang [5] the researcher introduced two methods for creating curve and surface models represented in Bézier and B-spline technique, and the reconstruction of surface in this research has been definition as parametric form terms. Given a known set of data on a surface, determine the defining the polygon net for a Bézier or B-spline surface are the best represented interpolates or approximates a set of known surface data.

Gerald [6] in this paper the researchers verified when boundary curve is given the surface is interpolated. The discrete bilinearly blended Coons patch is defined as the solution of a linear system. And also discuss triangular coons patches and point out the connections of the rectangular case. This idea is generalized for producing better shape than the Coons patch.

Seungyong [7] the research introduce algorithm to approximation and interpolation the scatter data. The $\mathrm{C}^{2}$ surface continuity are compute through multilevel B-Spline by a set of points has nonuniform spaced. The algorithm is based on Bspline approximation. A B-spline control lattice is efficiently determined by minimizing a local approximation error for each control point.

Fredrik [8] the research described the theoretical and mathematically about graphical and geometrical represented of curves and surfaces. These techniques considered most interesting terms those studies in many different areas theory and practical, and the two-popular technique (Bezier and B-Spline) representations the curve and surface these techniques.

Musra [9] presented free form surface by applying the main B-Spline functions for building curves and surface. Surface and curves, concerning the research were built by using a set of the required Algorithms and executed on MATLAB 6 program. The stages of building are as follows: first stage deals in the uniform B-Spline surface and curves for the second, third grade there by the direct method is applied in generating the B-Spline surface and curves through inserting the control points and the indirect method (inverse Algorithm) through inserting the correlation points between the parts of the curve. Concerning the second stage, Non-uniform B-Spline functions, are used for the second and third grade to generate the free form surface.

\section{Proposed of Developed Approach}

In this research, a new approach introduced to redesign and construction of freeform surface. as illustrate in Figure 1 Overflow of the developed approach includes 3D CAD design, Data extraction from data exchange file, Calculation of the mathematical model based on non-uniform BSpline technique, and finally Represent the freeform with multi patches surfaces. This approach gives the designers more freedom and flexibility in design freeform shape, the cause return to its mathematical model that is able to represent surface has high degree of complexity.

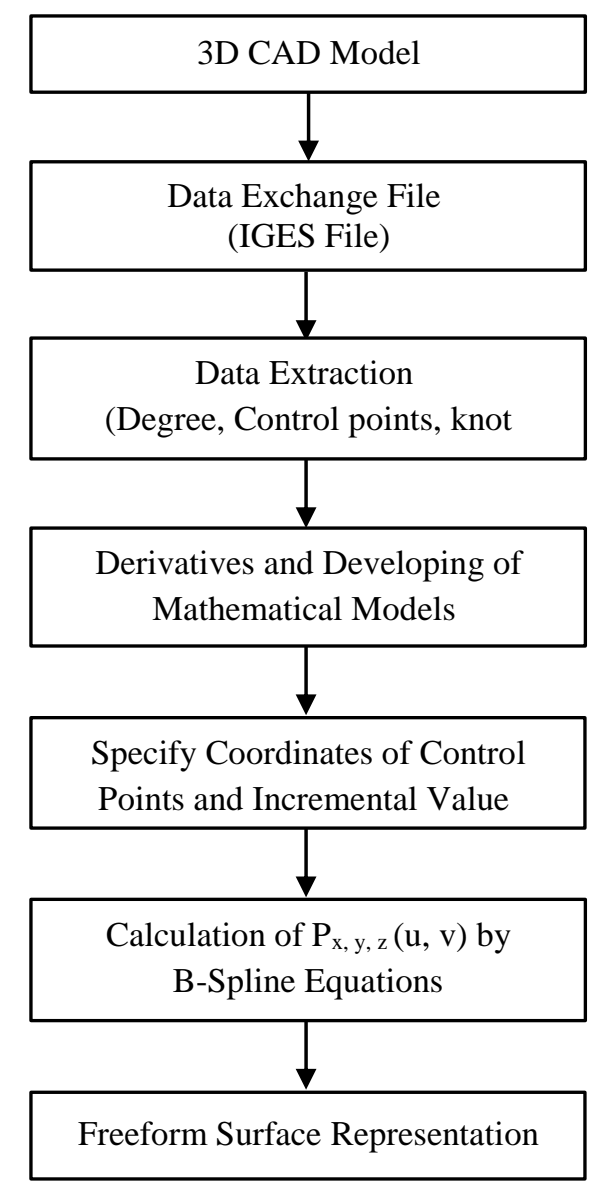

Figure 1: Overflow of Proposed Approach 


\section{3D CAD Model Design}

The part design in this research is presented by CAD software and as a solid model by using NonUniform Non-Rational B-Spline technique as design tools. 3D CAD model has been designed by using CAD software (Solid Work 2014). The solid model of the part design consists of freeform surface. The CAD software generates the geometrical data of design model in the form of an (IGES) file format which is used as standard and general format which able the proposed methodology to communicate with the different CAD/CAM. In Figures 2-3 will be show the 3D CAD model design and their IGES format file.

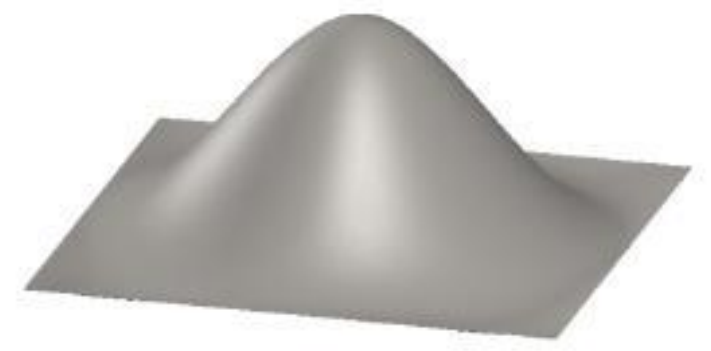

Figure 2: 3D CAD Model by Solid Works Software

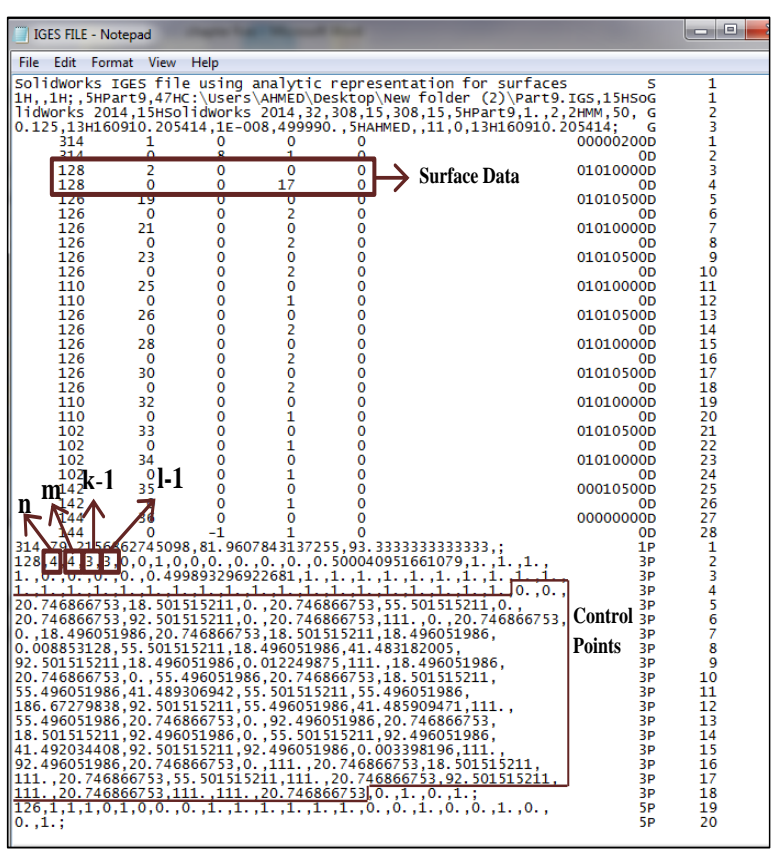

Figure 3: The IGES File Format of 3D CAD Model

\section{Mathematical Representation of B-Spline Curve and Surface}

\section{B-Spline curve}

The difference between B-Spline curves, Hermite or Bezier curve in that B-Spline curve it usually consists of more than one curve segment. All segments are represented and affected by a set of control points, which are the coefficient of BSpline basis function polynomials. The total number of control points it has nothing to do with degree of curve, so there is a possibility to manipulate and change in shape that do not propagate beyond one or only few local segments. The mathematical form of $\mathrm{B}$-Spline curve is represented by [13]:

$P(u)=\sum_{i=0}^{n} P_{i} N_{i, k}$

Where:

$P_{i}$ : Control points.

$N_{i, k}$ : Appropriate basis function for B-Spline

representation

$k \quad$ : Curve degree.

The basis function $\left(N_{i, k}\right)$ is defined by following recursive equation:

$$
\begin{array}{ccc}
N_{i, 1}(u)=1 & \text { If } & t_{i} \leq u \leq t_{i+1} \\
=0 & \text { otherwise }
\end{array}
$$

Where:

$t_{i}$ : knot values, and a set of knot values called knot vectors $(\mathrm{T})$.

The function to determine the knot vectors related with number of control points and degree of polynomials curve as show in the following equation:

$n+k+1=T$

The knot vector has a significant influence on the blending function $N_{i, k}(t)$ these knot vectors can be classified as periodic and non-periodic knots. Since the knot vectors influence the shape of the B-spline, it can be said, in general, that B-Spline curves are classified into uniform B-spline and non-uniform B-Spline curves. [11]

$T=\left[\begin{array}{lllllll}0 & 1 & 2 & 3 & 4 & 5 & 6\end{array}\right]$ Uniform - spline Knot vector

$T=\left[\begin{array}{lllllll}0 & 0 & 0 & 1 & 2 & 2 & 2\end{array}\right]$ Non - Uniform - spine Knot vector

\section{Uniform B-Spline curve}

Many geometric modeling situations do not require the curve to pass through the end points. The uniform B-Spline curve is a piecewise polynomial curve defined by a set of control points which the curve ordinarily does not interpolate the end points. This method produces with a uniform or periodic knot vector, the basis function repeats itself identically over successive intervals of the parametric variable. Show Figure 5.

The final equation of Uniform B-spline curve when $\mathrm{k}=3$ are [14]:

$P_{i}(u)=\frac{1}{2}\left[(1-u)^{2} P_{i-1}+\left(-2 u^{2}+2 u+1\right) P_{i}+u^{2} P_{i+1}\right]$ 


\section{Non-Uniform B-Spline Curve}

The non-uniform B-spline is more general than the uniform or open B - splines, although it is not the most general type of this curve. It is obtained when the knot values are not equally spaced. [12]

The basis function defining one segment may differ from those defining another. This allows interpolating one or more of the control points. Show Figure 4

$$
\begin{aligned}
& t_{i}=0 \quad \text { if } \quad i<k \\
& t_{i}=i-k+1 \quad \text { if } \quad k \leq i \leq n \\
& t_{i}=n-k+2 \text { if } \quad i>n
\end{aligned}
$$

Where:

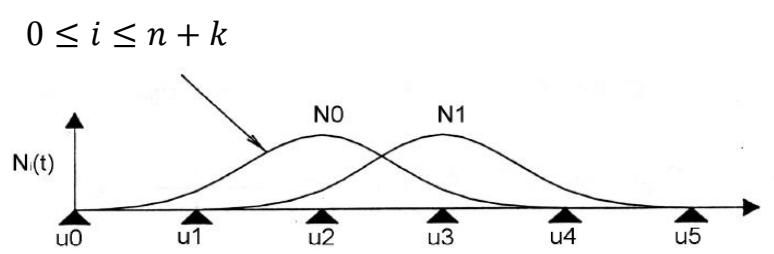

Figure 4: Cubic B-Spline Basis Function

\section{B-Spline Surface}

B-spline surfaces are an extension of B-spline curves. The most common kind of a B-spline surface is the tensor product surface. The surface basis functions are products of two univariate (curve) bases. The surface is a weighted sum of surface (two dimensional) basis functions. The weights are a rectangular array of control points. The following Eq. (7) shows a mathematical description of the tensor product B-spline surface.

$P(u, v)=\sum_{i=0}^{n} \sum_{i=0}^{m} p_{i j} N_{i, k}(u) N_{j, l}(v)$

Where:

$\mathrm{P}(\mathrm{u}, \mathrm{v})=\mathrm{B}$-spline surface as a function of two variables.

$p_{i j} \quad=$ control points.

$N_{i, k} \quad=i$ th basis function of order $\mathrm{k}$ as a function of $\mathrm{u}$.

$N_{j, l} \quad=j$ th basis function of order 1 as a function of $\mathrm{v}$.

For most computer aided design purposes, as in the case of the curve, $s(u, v)$ is a vector function of two parametric values $\mathrm{u}$ and $\mathrm{v}$. A mathematical description of this relationship is shown below in Eq. (8) [10].

$$
P(u, v)=\left\{\begin{array}{l}
x(u, v)=\sum_{i=0}^{n} \sum_{i=0}^{m} p_{x i j} N_{i, k}(u) N_{j, l}(v) \\
y(u, v)=\sum_{i=0}^{n} \sum_{i=0}^{m} p_{y i j} N_{i, k}(u) N_{j, l}(v) \\
z(u, v)=\sum_{i=0}^{n} \sum_{i=0}^{m} p_{z i j} N_{i, k}(u) N_{j, l}(v)
\end{array}\right.
$$

Where:

$\mathrm{x}, \mathrm{y}$ and $\mathrm{z}$ are coordinates in model space.

\section{Uniform B-Spline Surface}

When the knot vector is uniform except at its two ends, the open uniform B-spline is obtained and where knot values are repeated $k$ times. The following are simple examples:
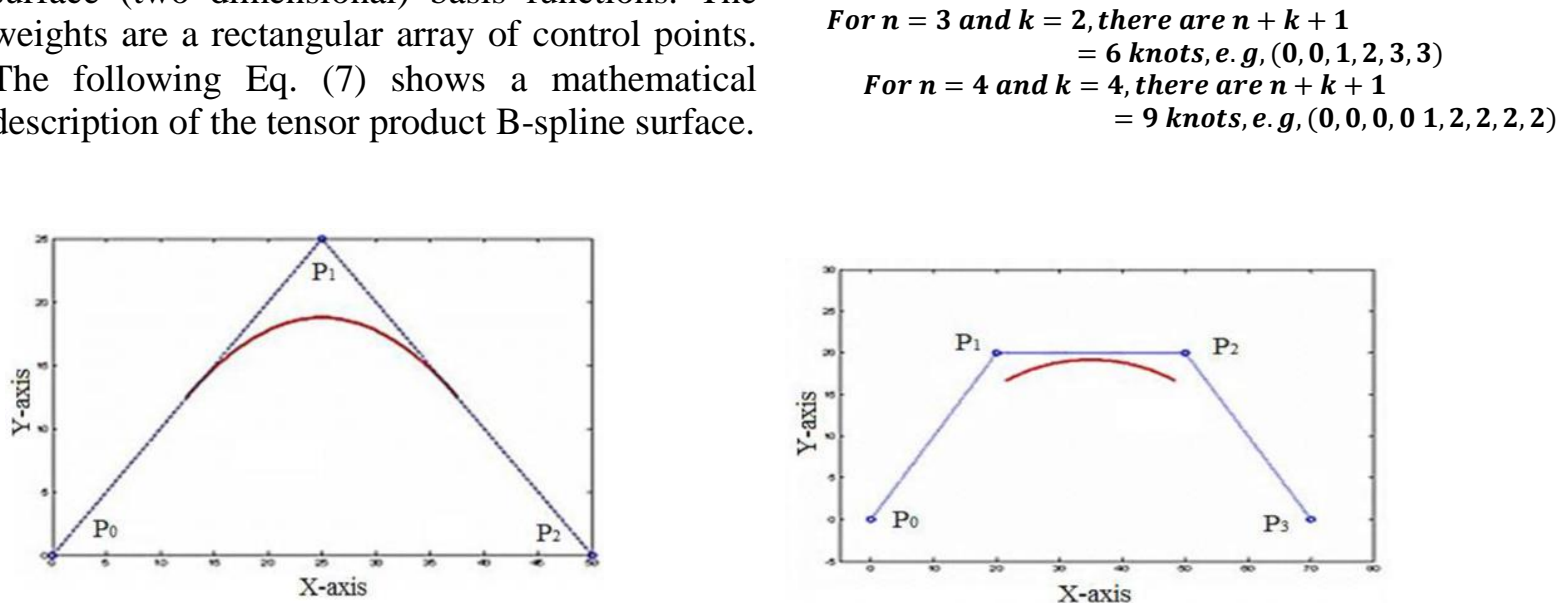

A: uniform B -spline Curve (2nd degree)

B: uniform B -spline Curve (3nd degree)

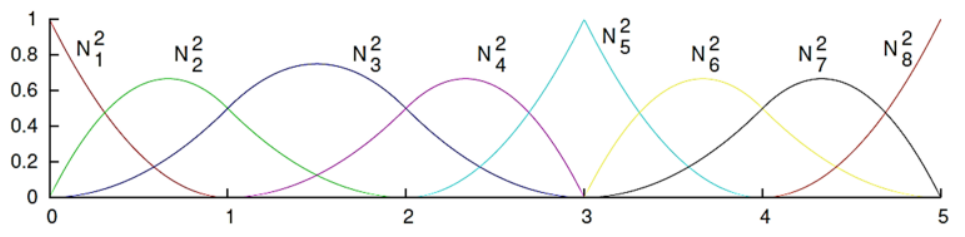

c: Basis functions of degree 2 on non-uniform.

Figure 5: $2^{\text {nd }}, 3^{\text {rd }}$ Degree of Uniform B-Spline Curve 
By using initial data for generating $3^{\text {rd }}$ degree of B- Spline surface with the aid of MATLAB (R2015a) software. Show Figure 6.

\section{Matrix Form:}

Similar to the B-spline curve, the B-spline surface is also a network of polynomial pieces. Each piece of the B-spline surface is a two dimensionally represented part of a surface or patch. As with a Bspline curve, each patch of a B-spline surface may be represented by a periodic relationship provided the knot spacing is uniform in each direction. This is a uniform B-spline surface. The bicubic case is described in matrix form by the following Equation:

$p_{i j}(u, v)=U M P M^{T} V^{T}$

Where:

$U=\left[\begin{array}{llll}u^{3} & u^{2} & u & 1\end{array}\right]$,

$V=\left[\begin{array}{llll}v^{3} & v^{2} & v & 1\end{array}\right]$

$M=\frac{1}{6}\left[\begin{array}{rrrr}-1 & 3 & -3 & 1 \\ 3 & -6 & 3 & 0 \\ -3 & 0 & 3 & 0 \\ 1 & 4 & 1 & 0\end{array}\right]$

$P=\left[\begin{array}{cccc}p_{i-1, j-1} & p_{i-1, j} & p_{i-1, j+1} & p_{i-1, j+2} \\ p_{i, j-1} & p_{i, j} & p_{i, j+1} & p_{i, j+2} \\ p_{i+1, j-1} & p_{i+1, j} & p_{i+1, j+1} & p_{i+1, j+2} \\ p_{i+2, j-1} & p_{i+2, j} & p_{i+2, j+1} & p_{i+2, j+2}\end{array}\right]$,

If the knot sequences are not uniformly spaced, then the surface is non-uniform. The basic functions would then have to be evaluated by the recursive relationship. The non-uniform patch Equation can be represented in matrix form. Eq. (12) shows this relationship for the bicubic case in the compacted form. [10].

$p_{i j}(u, v)=\left[\begin{array}{llll}N_{0,4}(u) & N_{1,4}(u) & N_{2,4}(u) & N_{3,4}(u)\end{array}\right]\left[\begin{array}{cccc}p_{i-1, j-1} & p_{i-1, j} & p_{i-1, j+1} & p_{i-1, j+2} \\ p_{i, j-1} & p_{i, j} & p_{i, j+1} & p_{i, j+2} \\ p_{i+1, j-1} & p_{i+1, j} & p_{i+1, j+1} & p_{i+1, j+2} \\ p_{i+2, j-1} & p_{i+2, j} & p_{i+2, j+1} & p_{i+2, j+2}\end{array}\right]\left[\begin{array}{c}N_{0,4}(v) \\ N_{1,4}(v) \\ N_{2,4}(v) \\ N_{3,4}(v)\end{array}\right]$

\section{Non-Uniform B-Spline Surface}

The non-uniform B-spline is more general than the uniform or open B - splines, although it is not the most general type of this curve. It is obtained when the knot values are not equally spaced. The basis function defining one segment may differ from those defining another. This allows interpolating one or more of the control points show Figure 7. The end knots of knot vector repeat with multiplicity $\boldsymbol{k}$, spacing the knots at unequal intervals of the parameter describes a non-uniform B-Spline curve, as well as the range of parametric variable $\boldsymbol{u}$ will be defined by the following equation: [13]

$0 \leq u \leq n-k+2$

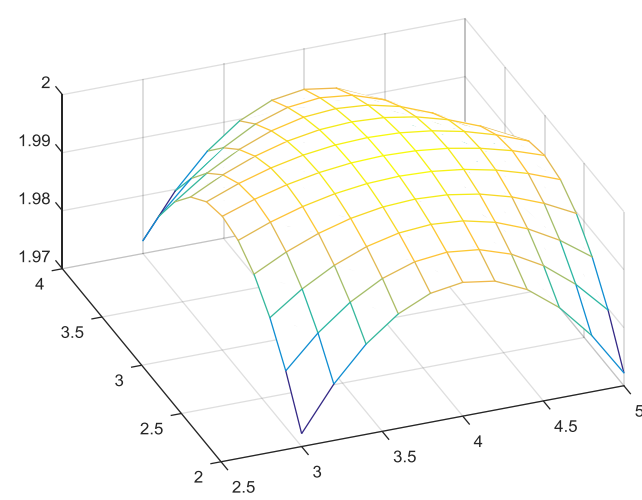

Figure 6: Uniform B-Spline Surface k=4

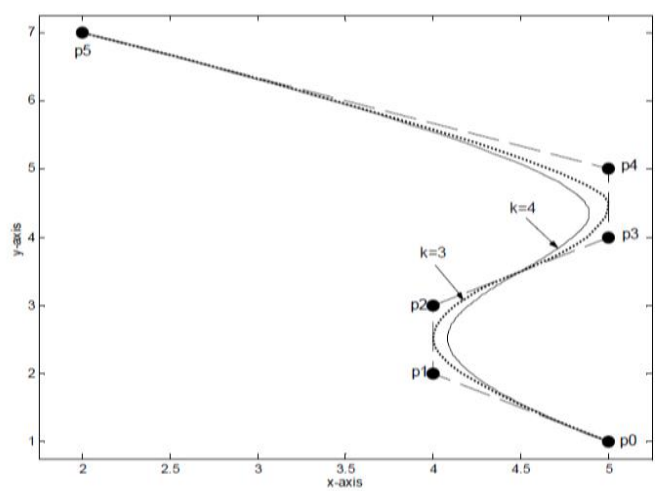

Figure 7: Non-uniform B-Spline Curve for 2nd \&3rd Degree.

\section{Design Freeform Surface Using Non- Uniform B-Spline Technique}

The non-uniform B-spline technique used to determine equation of the freeform surfaces, by mathematical description of the tensor product Bspline surface Eq. (7)

$P(u, v)=\sum_{i=0}^{n} \sum_{i=0}^{m} p_{i j} N_{i, k}(u) N_{j, l}(v)$

And to determine the basis function of the bicubic patch surface by Eq (3):

$N_{i, k}=\frac{\left(u-t_{i}\right) N_{i, k-1}(u)}{t_{i+k-1}-t_{i}}+\frac{\left(t_{i+k}-u\right) N_{i+1, k-1}(u)}{t_{i+k}-t_{i+1}}$

The following case study has $(\mathbf{n}=\mathbf{4}, \mathbf{k}=\mathbf{4})$, knot vector values from $t_{0}$ to $t_{n+k}$, (there are four more knots than control points), so the minimum number of knots is nine according Eq. (4):

$n+k+1=T$

The minimum control point number is four and parametric values $u$ will be from:

$t_{k-1}=t_{3}$ to $t_{n+1}=t_{5}$

The range of parametric variable (u) as Eq. (14):

$0 \leq u \leq n-k+2$

[ $0 \leq u \leq 2$ ] 
According the following condition will determine the knot vector $\mathbf{T}$ as Eq. (6):
$t_{i}=0$
if $\quad i<k$
$t_{i}=i-k+1 \quad$ if $\quad k \leq i \leq n$
$t_{i}=n-k+2$ if $i>n$

And by repeating of end knots with multiplicity $\boldsymbol{k}$, knot vector will be:

$T=\{0,0,0,0,1,2,2,2,2\}$

Using the Eq. (2) B-spline variables were compensated and set of equations were obtained for each $\boldsymbol{k}$, as a result for compensating these equations between each other we got the final equations which is:

$$
\begin{aligned}
& N_{0,4}=\left(1-3 u+3 u^{2}-u^{3}\right) N_{3,1} \\
& N_{1,4}=\left(3 u-\frac{9}{2} u^{2}+\frac{7}{4} u^{3}\right) N_{3,1+}\left(2-3 u+\frac{3}{2} u^{2}-\frac{1}{4} u^{3}\right) N_{4,1} \\
& N_{2,4}=\left(\frac{3}{2} u^{2}-u^{3}\right) N_{3,1+}\left(-2+6 u-\frac{9}{2} u^{2}+u^{3}\right) N_{4,1} \\
& N_{3,4}=\frac{1}{4} u^{3} N_{3,1+}\left(2-6 u+6 u^{2}-\frac{7}{4} u^{3}\right) N_{4,1} \\
& N_{4,4}=\left(-1+3 u-3 u^{2}+u^{3}\right) N_{4,1}
\end{aligned}
$$

From the equations above the two matrices will be extracted for tow patches as follows:

$\mathrm{Mb1}=\frac{1}{4}\left[\begin{array}{cccc}-4 & 7 & -4 & 1 \\ 12 & -18 & 6 & 0 \\ -12 & 12 & 0 & 0 \\ 4 & 0 & 0 & 0\end{array}\right]$,

$\mathrm{Mb} 2=\frac{1}{4}\left[\begin{array}{cccc}-1 & 4 & -7 & 4 \\ 6 & -18 & 24 & -12 \\ -12 & 24 & -24 & 12 \\ 8 & -8 & 8 & -4\end{array}\right]$

Under those final basis functions of surface model were obtained two variable basis functions $N_{3,1}, N_{4,1}$ and the matrix forms of those variable functions are:

$P_{i}(u) U M b_{i} P_{i-1} M b_{i}^{T} V^{T}$

Where:

$M b_{i}=$ Matrices of two variable basis function.

The freeform surface of $(n=4, k=4)$ will be contained multi patches to form it and the matrix form of variable basis function $N_{3,1}$ (patch one) will be:

$$
P_{1}(u)=U M b_{1} P_{i-1} M b_{1}^{T} V^{T}
$$

The final matrix will be as follows:

$P_{1}(u)=\frac{1}{4}\left[\begin{array}{llll}u^{3} & u^{2} & u & 1\end{array}\right]\left[\begin{array}{cccc}-4 & 7 & -4 & 1 \\ 12 & -18 & 6 & 0 \\ -12 & 12 & 0 & 0 \\ 4 & 0 & 0 & 0\end{array}\right]\left[\begin{array}{l}p_{0} \\ p_{1} \\ p_{2} \\ p_{3}\end{array}\right]$

The control points $\left(P_{i}\right)$

$P_{x}=\left[\begin{array}{llll}0 & 18.5015 & 55.5015 & 92.5015 \\ 0 & 18.5015 & 55.5015 & 92.5015 \\ 0 & 18.5015 & 55.5015 & 92.5015 \\ 0 & 18.5015 & 55.5015 & 92.5015\end{array}\right]$

$P_{y}=\left[\begin{array}{cccc}0 & 0 & 0 & 0 \\ 18.4961 & 18.4961 & 18.4961 & 18.4961 \\ 55.4961 & 55.4961 & 55.4961 & 55.4961 \\ 92.4961 & 92.4961 & 92.4961 & 92.4961\end{array}\right]$

$P_{Z}=\left[\begin{array}{cccc}20.7469 & 20.7469 & 20.7469 & 20.7469 \\ 20.7469 & 0.0089 & 41.4832 & 0.0122 \\ 20.7469 & 41.4893 & 186.6728 & 41.4859 \\ 20.7469 & 0 & 41.4920 & 0.0034\end{array}\right]$

For the matrix form of variable basis function $N_{4,1}$ (patch two) will be:

$P_{1}(u)=U M b_{2} P_{i-1} M b_{2}^{T} V^{T}$

And Matrix Form will be as follows:

$P_{2}(u) \frac{1}{4}\left[\begin{array}{llll}u^{3} & u^{2} & u & 1\end{array}\right]\left[\begin{array}{cccc}-1 & 4 & -7 & 4 \\ 6 & -18 & 24 & -12 \\ -12 & 24 & -24 & 12 \\ 8 & -8 & 8 & -4\end{array}\right]\left[\begin{array}{l}p_{1} \\ p_{2} \\ p_{3} \\ p_{4}\end{array}\right]$

The control points $\left(P_{i}\right)$

$P_{x}=\left[\begin{array}{llll}18.5015 & 55.5015 & 92.5015 & 111 \\ 18.5015 & 55.5015 & 92.5015 & 111 \\ 18.5015 & 55.5015 & 92.5015 & 111 \\ 18.5015 & 55.5015 & 92.5015 & 111\end{array}\right]$

$P_{y}=\left[\begin{array}{cccc}18.4961 & 18.4961 & 18.4961 & 18.4961 \\ 55.4961 & 55.4961 & 55.4961 & 55.4961 \\ 92.4961 & 92.4961 & 92.4961 & 92.4961 \\ 111 & 111 & 111 & 111\end{array}\right]$

$P_{z}=\left[\begin{array}{cccc}0.0089 & 41.4832 & 0.0122 & 20.7469 \\ 41.4893 & 186.6728 & 41.4832 & 20.7469 \\ 0 & 41.4920 & 0.0034 & 20.7469 \\ 20.7469 & 20.7469 & 20.7469 & 20.7469\end{array}\right]$ 


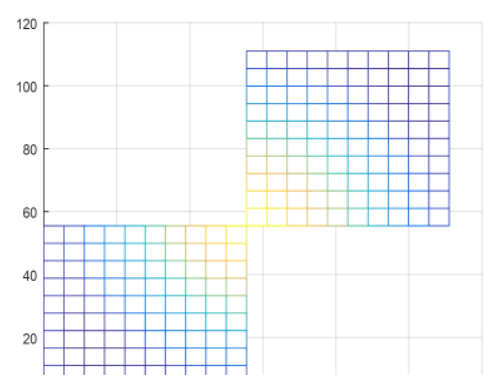

a) Patch $1 \& 2$ of B-Spline

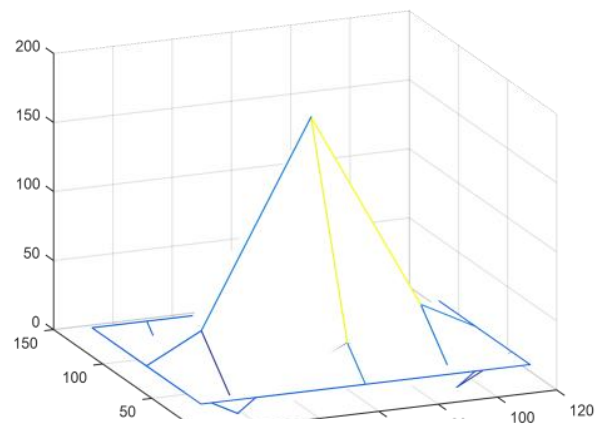

c) Control Points Network

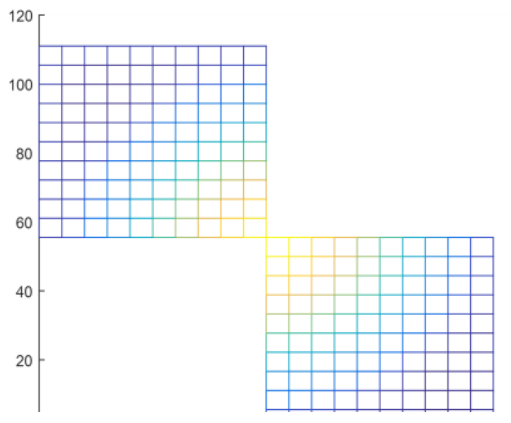

b) Patch $3 \& 4$ of B-Spline Surface

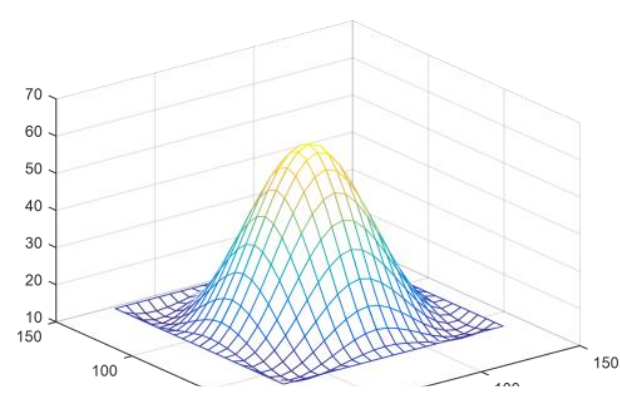

d) Four Patches of B-Spline Surface

Figure 8: Multi patches of B-Spline Surface.

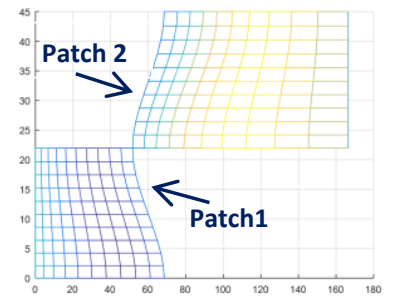

a) Patch $1 \& 2$ of B-Spline Surface

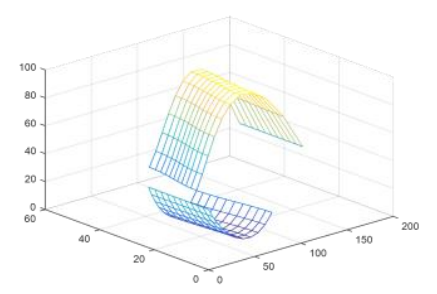

c) Patch $1 \& 2$ of B-Spline Surface with $\mathrm{Z}$ axis

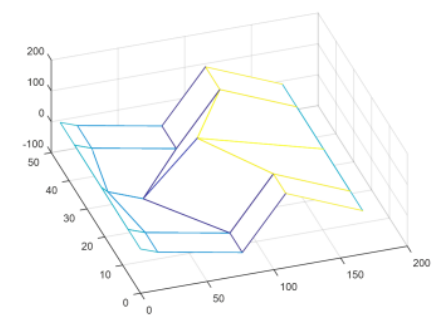

e) Control Points Network

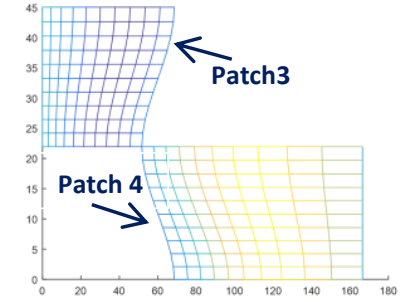

b) Patch $3 \& 4$ of B-Spline Surface

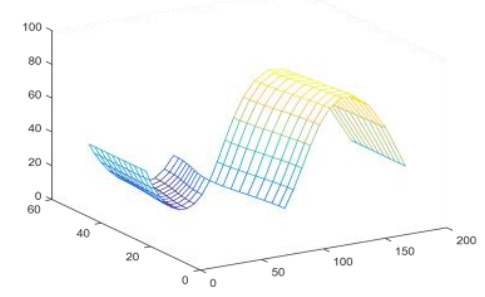

d) Patch $3 \& 4$ of B-Spline Surface with $\mathrm{Z}$ axis

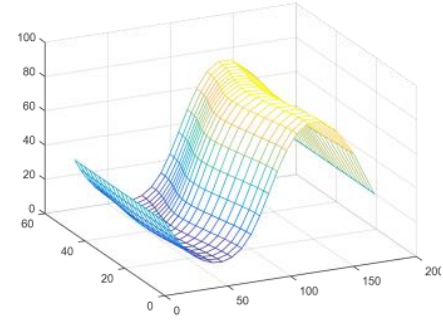

f) For Patches of B-Spline Surface

Figure 9: Multi patches of B-Spline Surface 
The mathematical representation of the surface by non-uniform technique for $(n=4, k=4)$ with aid MATLAB software (R 2015a) will be in Figures 8-9:

\section{Discussion}

For Non-Uniform B-Spline Surface $(n=4),(k=4)$ four patches which synthesis the surface, each patch has four control points according degree of the surface and its order (k-1), patch 1 and 2 it's the main patch of the surface each one has matrix and parametric value in both direction $\mathrm{u}, \mathrm{v}$ as show in the Figure (8a), patch 3and 4 it's the opposite patch for 1and 2 in control points and matrix as show in Figure (8b). Thus, the Non-Uniform BSpline surface has five control point construction the polygon network of control points of the surface as show in Figure (8c). The control points distribution for each patch according Eq. (17), (19). The patches of the surface may be increasing depending on $\mathrm{n}, \mathrm{k}$ variables of the non-uniform $\mathrm{B}$ Spline surface. The basis function Eq. (3) that used to derivatives the matrices of the B-Spline surface is the best methods which help in represent the surface in mathematical model to obtain to geometric data of the surface.

\section{Conclusion}

In this research the algorithm has been implemented for freeform B-Spline surface of 3D CAD Models and represented in mathematically. Some of interested conclusions summarized in the followings:

- It has been proved that the Non-Uniform BSpline is a suitable technique that used to represent the complex shapes has freeform surfaces by using control points network that generally lies outside the actual surface and the multi patches construction of the surfaces.

- The symbolic computation of B-Spline surface, as a tool which play an important role in many $\mathrm{CAD} / \mathrm{CAM}$ areas. It's applied to obtain the meaningful regions from low level B-Spline surfaces.

- IGES file format is a suitable to obtain the important parameters and geometric data such control points and degree of the surface that necessary to implement the algorithm.

- The first and second derivatives of B-Spline surface equation is necessary computation part to obtain all geometrical data of the freeform surface which are used later in the proposed methodology. - The multi patches that necessary to synthesis the Non-Uniform B-Spline surface consider important property. While giving sufficient freedom for designer in modeling and design freeform surface have more complexity.

\section{Acknowledgement}

This Work is supported by the Industrial Department of Production and Metallurgy Engineering, University of Technology; the authors also gratefully acknowledge the support of the technology University Especially Engineering Department to contribute to the provision of scientific research.

\section{Reference}

[1] T. W. Sederberg, "Computer Aided Geometric Design Course Notes, Chapter 6 "B-Spline Curves," September 28, 2016.

[2] J. I, Hoffman, "Constrained shape modification of cubic B-spline curves by means of knots,' ComputerAided Design Journal, Vol.36, PP. 437-445, 2003.

[3] S.S. Randhawa, J. S. Saini, "Tool Path Generation for Free-Form Surfaces Using B-Spline Surface," Vol.81, PP. 22-1859, Springer India 2014.

[4] P. Mach, "NURBS for Nerds Surface in Computer Graphics and Manufacturing," Mathematics 4899, 2004.

[5] X. Guo, "Reconstruction of Parametric Curves and Surface Using an Adaptive Basis Function Network" M.Sc. Thesis, University of Western Ontario, 1998.

[6] G. Farin, D. Hansford, "Discrete Coons Patches," Computer Aided Geometric Design, Vol.23, No.2, pp.691-700, 1999.

[7] S. Lee, G. Wolberg and S. Y. Shin "Scattered Data Interpolation with Multilevel B-splines," IEEE Transactions on Visualization and Computer Graphics, Vol.3, No.3, 1997.

[8] F. Anderson, "Bezier and B-Spline Technology," 2003, Available: http://www.cs.umu.se/education/Rapporter/461.pdf

[9] H.K. Musra "Development of the Free Form Surface Construction Approach by Using Computer," M.Sc. Thesis, University of Technology, 2002.

[10] K. Bindiganavle, "An Optimal Approach to Geometric Trimming of B-Spline Surfaces," Mechanical Engineering, Virginia Polytechnic Institute and State University Blacksburg, 2000.

[11] M. E. Mortenson "Geometric Modeling" John Wiley and sons Inc., 1997.

[12] D. Salomon, "Curves and Surfaces for Computer Graphics," Department of Computer Science California State University, Northridge, Springer Science, 2006.

[13] M.M. Abdul-Razaq, "Constructing of MultiPatches B-spline Surfaces," Production and Metallurgy Engineering Department, University of Technology, Eng. And Tech. Journal, Vol. 33, No. 4, 2015.

[14] A.S. Bedan, "Automatic surface generation from wire frame data in CAD applications," MSc. Thesis, Production and Metallurgy Engineering University of Technology, Baghdad, 2006. 


\section{Author(s) biography}

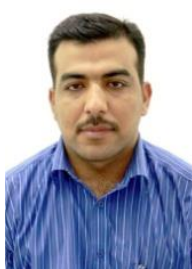

Amjad. B. Adulghafour, Lecturer,

Production Engineering and Metallurgy

Department University of Technology,

Baghdad, Iraq,

Previous Publications: Engineering and

Technology Journal

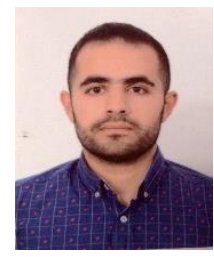

Ahmed. T. Hassan, Student, Master degree, Production Engineering and Metallurgy Department University of Technology, Baghdad, Iraq

Member of the Instituted of Electrical and Electronic Engineering (IEEE)

Previous Publications: Instituted of Electrical and Electronic Engineering Journal 